This case underscores the fact that in this patient population, lymphoma may have atypical clinical or radiographic features. NHL should be considered in the differential diagnosis of any patient with AIDS who presents with unilateral orbital signs, such as proptosis or motility disturbances. With improvements in diagnosis and treatment of this disease, short and long term prognosis may improve.

1 Khojasteh A, Reynolds RD, Khojasteh CA. Malignant lymphoreticular lesions in patients with immune disorders resembling acquired immunodeficiency syndrome (AIDS): review of 80 cases. South $M e d \mathcal{F} 1986 ; 79: 1070-5$.

2 Schuman JS, Orellana J, Friedman AH, Teich SA. Acquired immunodeficiency syndrome (AIDS). Surv Ophthalmol 1987; 31: 384-410.
3 Henderson JW, Farrow GM. Orbital tumors. 2nd ed. New York: Brian C Decker, 1980; 70-4.

4 Rosenberg SA, Diamond HD, Jaslowitz B, Craver LF. Lymphosarcoma: a review of 1269 cases. Medicine 1961; 40: 31-84.

5 Yeo JH, Jakobiec FA, Abbot GF, Trokel SL. Combined clinical and computed tomographic diagnosis of orbital lymphoid tumors. Am $\mathcal{F}$ Ophthalmol 1982;94: 235-45.

6 Mansour AM. Orbital findings in acquired immunodeficiency syndrome. Am f Ophthalmol 1990; 110: 706-7.

7 Antle CM, White VA, Horsman DE, Rootman J. Large cell orbital lymphoma in a patient with acquired immunodeficiency syndrome. Ophthalmology 1990; 97: 1494-8.

8 Turok DI, Meyer DR. Orbital lymphoma associated with acquired immunodeficiency syndrome. Arch Ophthalmol 1992; 110: 610-1.

9 Jabs DA, Green WR, Fox R, Polk BF, Bartlett JG. Ocular manifestations of acquired immune deficiency syndrome. manifestations of acquired imm

10 Ziegler JL, Beckstead JA, Volberding PA, Abrams DI, Levine $A M$, Lukes RJ, et al. Non-Hodgkin's lymphoma in 90 homosexual men: relation to generalized lymphadenopathy and the acquired immunodeficiency syndrome. $N E n g f \mathcal{f} M$ 1984; 311: 565-70.

\title{
Ocular pulse measurements to assess pulsatile blood flow in carotid artery disease
}

\author{
K G Claridge, C B James
}

Patients with carotid artery stenosis frequently present with symptoms associated with embolic episodes or, more rarely, with chronic ocular ischaemia, the varied signs of which include hypotensive retinopathy, uveitis, rubeosis, corneal decompensation, and cataract. ${ }^{1}$ Carotid Doppler ultrasound scanning is the preferred test to detect those patients with severe stenosis who might benefit from vascular surgery and, in the postoperative period, to monitor increased carotid perfusion. ${ }^{2}$ Assessment of ocular perfusion is not routinely performed. We report non-invasive measurement of pulsatile ocular blood flow (POBF) using the Langham pneumotonometer ${ }^{34}$ in two patients undergoing carotid artery surgery.

\section{Case reports}

CASE 1

A 72-year-old man presented with a 4 month history of left sided amaurosis fugax relieved by stooping. He had intermittent claudication and took oxpentifylline. His acuity was $6 / 9$ in the right eye and counting fingers in the left. The right eye was normal apart from a low central retinal artery perfusion pressure revealed by digital compression of the globe. The left eye showed low grade anterior uveitis with rubeosis, an unreactive dilated pupil, widespread peripheral fundal haemorrhages, and a very low central retinal artery perfusion pressure, pulsating with the slightest pressure on the globe. After intravenous acetazolamide the left vision improved to $6 / 18$. There were bilateral carotid bruits, and absent pulses in the right arm and distal to both femoral arteries.

Fluorescein angiography of the left eye confirmed slow flow retinopathy, with a prolonged arm to retina time of 19 seconds (normal $<15$ seconds) and delayed arteriovenous transit, some retinal veins never filling completely. Doppler ultrasound demonstrated reduced flow velocity in the right common, external, and internal carotids; no flow in the left carotid system; reverse flow in the right vertebral; and increased velocity in the left vertebral.

Digital subtraction angiography (DSA) was consistent with these findings demonstrating an occlusion of the right innominate and left common carotid arteries at their origins. Intracranial flow was solely from the left vertebral artery. Surgery revealed a tight atheromatous stenosis in the left internal carotid artery extending from the bifurcation. Following left subclavian to right common and internal carotid Dacron grafting there were no further episodes of amaurosis fugax though the clinical signs were unchanged.

Ocular pulse measurements and systemic blood pressure were recorded before, and 5 days after surgery, in the supine position (Fig 1). The POBF increased in the postoperative period by more than four times in the right eye and rose from being unrecordable to almost $200 \mu \mathrm{l} / \mathrm{min}$ in the left eye (Table 1).
Musgrove Park, Taunton,

Accepted for publication

8 October 1993 


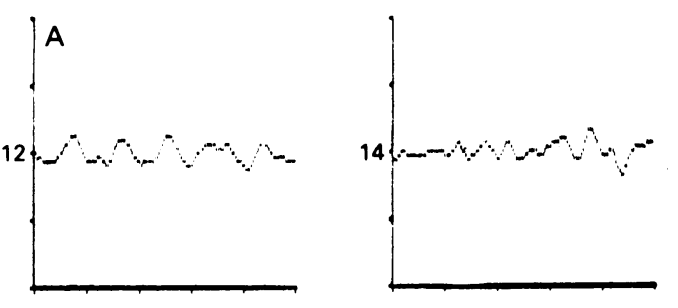

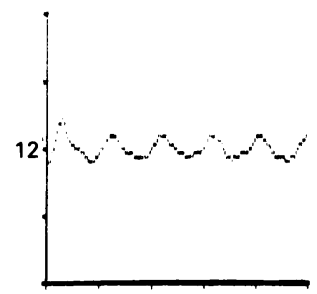

Right eye
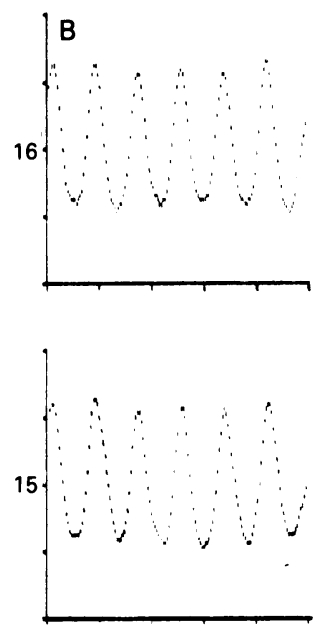

Right eye

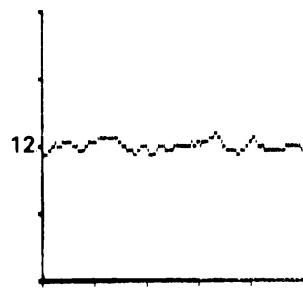

Left eye
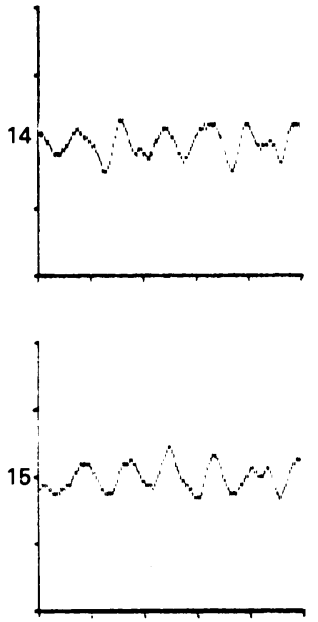

Left eye
Figure 1 Langham pneumotonometer intraocular pressure pulse recordings $(A)$ before and $(B) 5$ days after surgery in case 1 .

\section{CASE 2}

A 55-year-old man presented with a history of light induced amaurosis fugax followed, just before admission, with complete loss of the lower visual field in the right eye. Visual acuity was $6 / 5$ in both eyes, anterior segment examination was normal but funduscopy revealed a right sided swollen disc with peripheral haemorrhages and cotton wool spots. Digital compression of both globes showed a low central retinal artery perfusion pressure. There were bilateral carotid bruits and a cardiac murmur suggestive of aortic

Table 1 Changes in intraocular pressure (IOP), pulse amplitude $(P A)$, pulsatile ocular blood flow $(P O B F)$, heart rate $(H R)$ and mean systemic blood pressure $(B P)$, before and after carotid surgery

\begin{tabular}{|c|c|c|c|c|c|c|c|c|}
\hline & \multicolumn{4}{|l|}{ Case 1} & \multicolumn{4}{|l|}{ Case 2} \\
\hline & \multicolumn{2}{|c|}{ Right eye } & \multicolumn{2}{|l|}{ Left eye } & \multicolumn{2}{|c|}{ Right eye } & \multicolumn{2}{|c|}{ Left eye } \\
\hline & Pre-op & Post-op & Pre-op & Post-op & Pre-op & Post-op & Pre-op & Post-op \\
\hline $\begin{array}{l}\mathrm{IOP}(\mathrm{mm} \mathrm{Hg}) \\
\mathrm{PA}(\mathrm{mm} \mathrm{Hg}) \\
\mathrm{POBF}(\mu \mathrm{l} / \mathrm{min}) \\
\mathrm{HR}(\mathrm{beats} / \mathrm{min}) \\
\mathrm{BP}(\mathrm{mm} \mathrm{Hg})\end{array}$ & $\begin{array}{c}12 \cdot 0 \\
0 \cdot 4 \\
118 \\
67 \\
89\end{array}$ & $\begin{array}{c}15 \cdot 7 \\
2 \cdot 2 \\
547 \\
72 \\
85\end{array}$ & $\begin{array}{l}12 \cdot 5 \\
- \\
-\end{array}$ & $\begin{array}{c}14 \cdot 5 \\
0.7 \\
173\end{array}$ & $\begin{array}{c}16 \cdot 0 \\
1 \cdot 6 \\
285 \\
54 \\
90\end{array}$ & $\begin{array}{c}14 \cdot 4 \\
2 \cdot 7 \\
520 \\
52 \\
92\end{array}$ & $\begin{array}{c}16 \cdot 5 \\
1.6 \\
280\end{array}$ & $\begin{array}{c}19 \cdot 8 \\
4 \cdot 3 \\
758\end{array}$ \\
\hline
\end{tabular}

In case 1 the PA and POBF were unrecordable in the left eye preoperatively. regurgitation. Anterior ischaemic optic neuropathy with slow flow retinopathy was diagnosed and the patient was started on acetazolamide and topical timolol. Fluorescein angiography showed slow filling of the choroidal circulation with a delay in retinal filling, the venous circulation was still unfilled at 24 seconds. DSA was consistent with carotid Doppler studies and demonstrated tight stenosis of the left internal carotid near its origin, almost total occlusion of the left external carotid and on the right, total occlusion of the internal carotid and stenosis of the external carotid arteries.

After left carotid endarterectomy, there were no further episodes of amaurosis fugax. Although the peripheral haemorrhages and cotton wool spots disappeared, the right optic nerve became atrophic and the right inferior hemianopia persisted. However, fluorescein angiography indicated improved retinal filling, the entire circulation filling within 24 seconds. Subsequently right carotid endarterectomy was performed and postoperative DSA showed the arteries to be functioning well.

Ocular pulse measurements were taken both before and after surgery on the left side, during which time the systemic and topical medications were unchanged (Table 1). There was a bilateral increase in POBF.

\section{Comment}

Both cases showed a dramatic increase in ocular pulse amplitude and the derived value for POBF after carotid artery surgery. Ocular perfusion, and therefore pulse amplitude and POBF, depends on the difference between mean ophthalmic artery pressure and IOP, so that an increase in the ophthalmic artery pressure or a fall in IOP will result in an increase in these parameters. There was in fact an increase in IOP without marked increase in blood pressure, following surgery on the ipsilateral side, implying a true increase in the ophthalmic artery pressure and therefore ocular blood flow following surgery. The bilateral increase in the ocular blood flow after unilateral surgery implies vascular patency within the circle of Willis.

The postural and light induced characteristics of the episodes of visual loss in the respective patients, suggests an aetiology secondary to critical hypoperfusion rather than embolism. The absence of amaurosis fugax postoperatively provides clinical evidence for increased ocular perfusion. Despite this, chronic hypotensive retinopathy often has a poor prognosis, owing to irreversible retinal ischaemia.

Pneumotonometry is a convenient method for monitoring pulsatile ocular blood flow. A review of non-invasive techniques for assessing flow in carotid disease showed that the Langham pneumotonometer used in conjunction with a scleral cup, had a sensitivity of $93 \%$ and specificity of $89 \%$ in determining carotid stenosis of more than $75 \%,{ }^{6}$ and compared favourably with suction oculoplethysmography and orbital Doppler ultrasonography. Further data are required to determine whether derived values for POBF can predict the degree of carotid stenosis in a given case of ocular ischaemia. 
We thank Dr R Ross Russell for allowing us to report on his patients and Professor S E Smith for advice on the manuscript. The work was funded by the Iris Fund for Prevention of Blindness and the Special Trustees of St Thomas's Hospital. The Langham Ocular Blood Flow System was purchased with a generous grant provided by Dispersa (UK) Ltd.

1 Dugan JD, Green WR. Ophthalmologic manifestations of carotid occlusive disease. Eye 1991; 5: 226-38.

2 Brown MM, Humphrey PRD. Carotid endarterectomy: recom- mendations for management of transient ischaemic attack and ischaemic stroke. BMF 1992; 305: 1071-4.

3 Langham ME, Farrell RA, O'Brien V, Silver DM, Schilder P. Blood flow in the human eye. Acta Ophthalmol 1989; 6 (suppl, 191): 9-13.

4 Silver DM, Farrell RA, Langham ME, O'Brien V, Schilder P. Estimation of pulsatile ocular blood flow from intracular pressure. Acta Ophthalmol 1989; 67 (suppl 191): 25-9.

5 Mills RP. Anterior segment ischemia secondary to carotid occlusive disease. 7 Clin Neuro-ophthalmol 1989; 9: $200-4$ 6 Langham ME, Preziosi TJ. Non-invasive diagnosis of mild to severe stenosis of internal carotid artery. Stroke 1984; 15: 614-21. 